\section{$\underset{\substack{\text { hommes } \\ \text { \& migrations }}}{ }$}

\section{Hommes \& migrations}

Revue française de référence sur les dynamiques

migratoires

$1315 \mid 2016$

Ondes de choc

\title{
Waed Bouhassoun
}

\section{François Bensignor}

\section{CpenEdition \\ Journals}

\section{Édition électronique}

URL : http://journals.openedition.org/hommesmigrations/3741

DOI : 10.4000/hommesmigrations.3741

ISSN : 2262-3353

\section{Éditeur}

Musée national de l'histoire de l'immigration

\section{Édition imprimée}

Date de publication : 1 juillet 2016

Pagination : 134-139

ISBN : 978-2-919040-36-0

ISSN : $1142-852 X$

\section{Référence électronique}

François Bensignor, «Waed Bouhassoun », Hommes \& migrations [En ligne], 1315 | 2016, mis en ligne le 02 janvier 2017, consulté le 15 septembre 2020. URL : http://journals.openedition.org/ hommesmigrations/3741 


\title{
MUSIQUES
}

\section{WAED BOUHASSOUN}

\author{
Par FRANÇOIS BENSIGNOR
}

$S_{\text {toms }}^{a}$ a voix ambre et velours peut éveiller les émotions les plus intimes sur une lamentation du djebel druze. Elle sait aussi transcender la poésie classique arabe comme autrefois l'illustre Asmahan, originaire du même terroir au Sud de la Syrie. Waed Bouhassoun, au jeu de oud délicat, est le garant d'un avenir pour la musique de Syrie, patrimoine en danger. Elle a choisi de vivre en France avant l'embrasement de son pays, mais demeure farouchement attachée à sa culture musicale. Son troisième album, La voie de la passion, vient de paraître sur le label Buda Musique. Le Festival de l'imaginaire l'associe à la programmation de sa $20^{\mathrm{e}}$ édition : nouvelle dimension dans le compagnonnage qui l'unit à la Maison des cultures du monde et à son équipe, Waed Bouhassoun propose en octobre 2016 un programme de concerts consacrés aux traditions musicales menacées au Proche et du Moyen-Orient*. Elle sera sur la scène du Théâtre des Abbesses en novembre. Rencontre.

Waed Bouhassoun a grandi dans un petit village druze proche de Soueïda au Sud de la Syrie. Depuis qu'elle a choisi de vivre en France, deux ans avant le début de la guerre civile, elle n'a cessé d'y retourner chaque année. Son brillant destin musical qui s'épanouit à partir de Paris, s'est d'abord dessiné au sein de sa famille, à laquelle elle est très attachée.

"Mon père enseigne la chimie et joue de la musique pour son plaisir. Un jour, il est rentré à la maison avec un oud. L'instrument était destiné à mon frère, mais comme il n'avait aucune envie d'apprendre, mon père me l'a confié. J'avais sept ans et c'est mon père qui m'a appris à jouer du oud. II m'a transmis de petites chansons traditionnelles de notre région, que je chantais d'oreille. Plus tard, j'ai abordé les répertoires des grandes dames du chant classique arabe de la première moitié $d u$ XXe siècle: Oum Kalthoum, Asmahan."

\section{Premier parcours musical en Syrie}

"Tout au long de ma scolarité, il y avait cet espace musical près de moi, sans que j'envisage d'en faire ma vie. Je suis quelqu'un qui parle peu. Mais, travers la conversation intime avec mon instrument, je pouvais traduire beaucoup de sentiments que je ne parvenais pas à exprimer dans la vie quotidienne. Jusqu'à 11 ans, je jouais à l'occasion des réunions familiales : le réveillon, les fêtes... Par la suite, dans le cadre scolaire, j'ai commencé à participer à des concours régionaux, qui se déroulaient dans toute la Syrie. C'était l'occasion de dormir hors de la maison. J'ai pu ainsi former très tôt ma personnalité musicale en dehors de la famille. À 18 ans, j'avais déjà circulé en Syrie pour mes activités musicales, soit en solo, soit avec de petits ensembles. " Après le bac, Waed Bouhassoun entre au Conservatoire de Damas. "Mon père m'a laissé choisir les études que je voulais poursuivre. Il a fallu préparer 


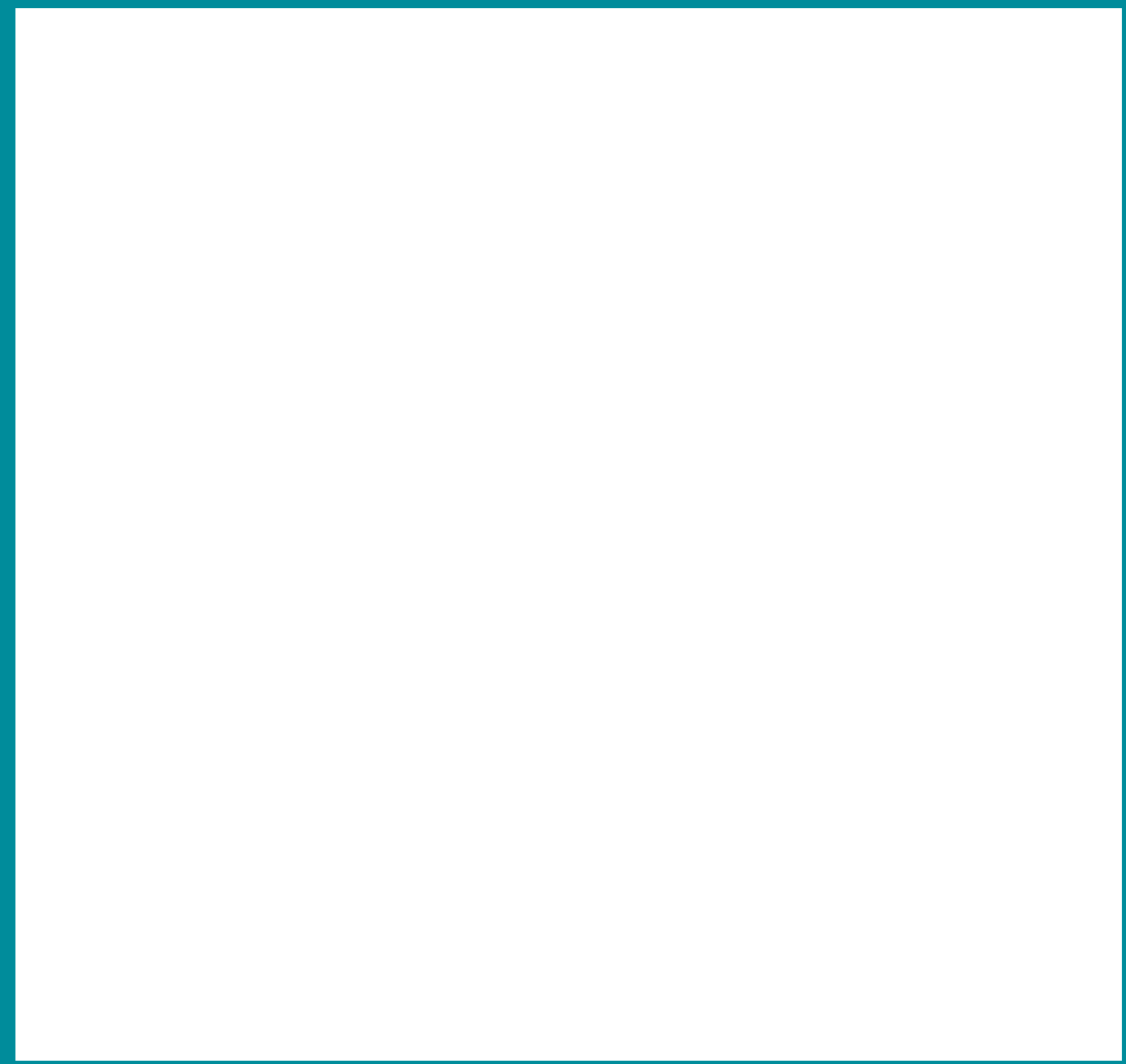

Waed Bouhassoun ๔ D.R.

mon concours d'entrée, qui reposait sur la musique occidentale, dont je n'avais aucune idée. Je me suis préparée seul à l'épreuve de chant lyrique: une opération complexe, parce qu'il fallait changer tout ce que je connaissais. Quand on chante dans un milieu de musique traditionnelle, on n'imagine pas la difficulté. J'ai quand même fait trois ans de chant classique lyrique, mais comme je ne m'y retrouvais pas, j'ai préféré arrêter et me spécialiser dans le jeu du oud." "

\section{Le lien avec la France}

Ainsi, c'est comme instrumentiste que Waed Bouhassoun est approchée par la tragédienne syrienne Maha Al-Saleh en 2004. Celle-ci prépare un monologue écrit pour elle par le dramaturge tunisien Ezzeddine Madani, programmé dans le cadre du Festival de l'imaginaire 2005. II retrace 


\section{MUSIQUES}

I'histoire de la reine Shajarat Ad Durr, contemporaine et victorieuse de Saint-Louis, qui régnait sur l'Égypte, Damas, Alep, Hama, Homs et le pays de Sham jusqu'au Golfe d'Aqaba, le Nord de l'Irak et du Yémen.

En France, les répétitions se déroulent dans la maison de Chérif Khaznadar, directeur-fondateur de la Maison des cultures du monde et du Festival de l'imaginaire. Aux yeux de l'assemblée d'artistes et de mélomanes, Waed Bouhassoun n'est alors qu'une joueuse de oud. Elle va la subjuguer quand on la sollicite pour chanter, interprétant une chanson de Oum Kalthoum. Durant la pièce, elle sera donc chanteuse, s'accompagnant au oud. Modeste, elle est cependant consciente de ses limites, estimant ne pas encore être suffisamment aguerrie au chant quand Chérif Khaznadar lui propose de donner un concert en solo.

Dès lors s'instaure un fructueux compagnonnage entre le maître originaire d'Alep qui a initié le public français aux musiques du monde et la jeune artiste. Souhaitant la programmer pour l'édition suivante du Festival de l'imaginaire, Chérif Khaznadar met Waed Bouhassoun en contact avec des musiciens d'Alep afin qu'elle prépare avec eux le répertoire de son futur concert. «Travailler avec des musiciens d'Alep était pour moi un peu comme un examen, car ils maitrisent des musiques magnifiques. Je suis allée régulièrement travailler avec eux, y passant quelques jours ou faisant l'aller-retour... Malheureusement, cela fait partie des libertés que l'on a perdues depuis... " La chanteuse entreprend un travail approfondi sur le son de sa voix. "Au conservatoire, le travail concernait la technique plutôt que la personnalité vocale. J'avais acquis la puissance, mais je trouvais ma voix un peu sèche. Avec les musiciens d'Alep, j'ai appris à faire passer par ma voix ce qui provient de l'âme. Ils mont conseillé d'écouter les grands chanteurs soufis arabes ainsi que les "munshid" qui chantent le Coran. Le faire m'a beaucoup appris sur la façon de prononcer chaque voyelle, chaque consonne de la langue arabe. Les "munshid", chanteurs de confrérie soufie, ont une technique pour faire résonner la voix dans tout le corps. Ils n'utilisent pas la colonne d'air verticale comme dans l'opéra. J'ai essayé plusieurs fois de suivre cet enseignement, mais les femmes n'y ont pas accès... Malgré cela, avec l'expérience, je parviens à faire passer des choses.

"Chérif Khaznadar m'a beaucoup conseillée et j'ai pu profiter de sa longue expérience. Depuis mon premier concert au Festival de l'imaginaire s'est posée la question de ma présence sur scène. En dehors de mon instrument et de ma voix, je dois entraîner le public dans mon univers. Je commence toujours mes concerts par une improvisation au oud. Cela me permet de me préparer moi-même et de créer une atmosphère. "

Invitée au Maroc en 2007 dans le cadre du Festival des musiques sacrées du monde de Fès, Waed Bouhassoun interprète des poèmes mystiques soufis et complète son répertoire avec des chants profanes. "Pour les poèmes, j'ai cherché à développer des mélodies qui servent la force de la poésie. Dans cette recherche, j'ai pu me rapprocher de la pensée des poètes. "Cet esprit traverse les pièces réunies dans son premier album solo, La voix de l'amour, publié en 2009 dans la collection de l'Institut du monde arabe et qui reçoit, cette même année, le « coup de cœur » de l'Académie Charles Cros.

"Quand je suis arrivée en France en 2010, Chérif Khaznadar a projeté l'enregistrement d'un second disque solo pour 2013. Il allait contenir mes propres compositions sur des poèmes mystiques et profanes. J'y ai ajouté trois poèmes d'Adonis. Ils me permettent d'exprimer ce que je ressens envers la Syrie. Durant son parcours d'exil en France, pendant les années 1950, Adonis a ressenti cette angoisse et cette inquiétude envers la syrie qu'il exprime à travers ses poèmes. " Dans ce deuxième album, L'âme du luth, Waed Bouhassoun parvient à trouver un style bien à elle, où se 
tion décernée à son premier album, permet à la chanteuse d'obtenir une bourse d'étude de deux ans. Ainsi, en 2010, après un premier séjour de deux mois à ses frais pour acquérir les premières notions de français à l'Alliance française, Waed Bouhassoun s'installe en France et entreprend un master d'ethnomusicologie à Nanterre. "Venir en France représentait pour moi un énorme défi : sur les plans de la langue, des démarches administratives pour obtenir des papiers, et des études. Commencer un master impliquait la lecture des ouvrages des grands anthropologues comme Claude LéviStrauss... C'était difficile et j'ai donné très peu de concerts entre 2010 et 2013. Pour moi, le plus compliqué est d'avoir à lire en français et en anglais sans passer par l'arabe. Et surtout de changer de manière de penser pour pouvoir écrire en français. Étudier ne me pose pas de problème, mais rédiger mon mémoire reste une difficulté. "

déploient à la fois une grande force et une sensibilité à fleur de peau. Comme le premier, cet album sera nommé " coup de cœur » par l'Académie Charles Cros.

\section{Le retour aux études}

À l'occasion de plusieurs invitations à se produire en marge de colloques consacrés à la musique dans le monde de l'islam, Waed Bouhassoun découvre l'ethnomusicologie, une discipline inconnue des universités syriennes. Sa proposition d'entamer un master consacré aux rituels funéraires des Druzes de Syrie séduit l'ethnomusicologue Jean Lambert, qui va devenir son directeur d'études. L'ambassade de France en Syrie, favorablement disposée grâce à la distinc-

\section{La rencontre avec Jordi Savall}

Quand éclate le conflit syrien en 2012, les liens se resserrent dans la communauté qui vit en France. Waed Bouhassoun sait qu'elle peut compter sur les familles qui l'entourent, notamment celles de Chérif Khaznadar et du poète Adonis. Mais aussi sur ses amis français, qui tous proposent de l'aider en cas de problème. Paradoxalement, le malheur de son pays va produire un effet de tremplin pour sa carrière d'artiste. Le maître catalan de la musique baroque Jordi Savall, qui s'est beaucoup intéressé aux relations entre l'Orient et l'Occident dans ses travaux de recherche musicale, veut rendre hommage à la Syrie. Son ensemble, Hespèrion XXI, va s'enrichir pour ce projet de deux artistes syrien : Waed Bouhassoun au chant et au oud, et l'un de ses anciens condisciples au conser- 
Waed Bouhassoun $\odot$ D.R.

vatoire de Damas, le flûtiste Moslem Rahal au ney. " Jouer avec Jordi Savall nous a permis de découvrir des cultures différentes, d'échanger avec d'autres musiciens venus du Maroc ou de Turquie. Entre Jordi et ses musiciens, il n'y a pas cette distance que l'on perçoit avec d'autres chefs d'or- chestres. Chacun est considéré sur un même plan d'égalité. Ce n'est pas un orchestre derrière une star. Une atmosphère très joyeuse règne entre les membres de l'orchestre qui est devenu ma deuxième famille. " Après avoir contribué à l'album Orient Occident II - Hommage à la Syrie (Alia Vox, 
2013), Waed Bouhassoun participe aux spectacles et aux deux nouveaux albums publiés par Jordi Savall : Ramon Llull (Alia Vox, 2016) et Granada (Alia Vox, 2016)

\section{La voix de la passion}

Cet automne est paru le troisième album de Waed Bouhasoun, La voix de la passion. Elle le consacre à cette poésie dialectale bédouine "nabatéenne », arrivée de la péninsule arabique jusqu'au Nord de la Syrie, qui fait l'objet de ses recherches et enquêtes de terrain pour son master. "L'accompagnement de cette poésie nécessite une sorte de bourdon, une note continue, qui est joué traditionnellement par un rebab. Le oud ne se prêtant pas à la production d'un son continue, j'ai choisi d'ajouter un autre instrument d'accom-

\section{Discographie :}

- La voix de la passion (Buda Musique, 2016).

- L'âme du luth (Buda Musique, 2014), " coup de cœur » de l'Académie Charles Cros.

- La voix de l'amour (Institut du Monde Arabe/ Harmonia Mundi, 2009), " coup de cœur » de l'Académie Charles Cros.

\section{Avec Jordi Savall :}

- Granada (Alia Vox, 2016).

- Ramon Llull (Alia Vox, 2016).

- Orient-Occident - Hommage à la Syrie (Alia Vox, 2013).

\section{Festival de l'Imaginaire 2016 :} www.festivaldelimaginaire.com/Programme

\section{Carte blanche à Waed Bouhassoun :}

- Les 13 et 14 octobre à l'Église Saint-Roch, l'Ensemble musical ecclésiastique levantin pagnement, la flûte ney, interprétée par mon ami le joueur de ney syrien Moslem Rahal. Dans mes recherches, j'ai constaté que les fruits de la transmission orale sont très particuliers. II est difficile à une personne qui n'a pas reçu un patrimoine oral dès la petite enfance de le reproduire. J'y travaille depuis 2014, en écoutant beaucoup afin de trouver la bonne prononciation des mots. "Entre parlée et chantée, cette poésie possède une grande musicalité. Certains poèmes servaient à encourager les chameaux à accélérer leur marche. On sent alors ce mouvement, comme un bateau fendant sur les sables du désert. D'autres poèmes exhortent à la guerre, chantent les victoires. Les poèmes de lamentation sont particulièrement poignants. L'imagination joue un rôle important dans la réception de ces poèmes. Ils portent en eux des notions de liberté qui me touchent : la liberté de penser, la liberté de circuler. »

de l'université Antonine (Liban) interprétera des cantiques, litanies et improvisations vocales des Églises syriaque orthodoxe, maronite, grecque orthodoxe, grecque catholique et copte.

- Le 15 octobre à l'Institut du monde arabe, l'Ensemble Goussan présentera le répertoire des anciens bardes et troubadours d'Arménie.

- Le 16 octobre à l'Institut du monde arabe, les artistes syriens Mona Issa et Bashar Abu Hamdan proposeront respectivement deux formes différentes de poésie dialectale chantée dans deux régions montagneuses. L'une vient du djebel Ansariyeh à l'ouest de la Syrie, l'autre du djebel Druze, porte du désert qui s'étend du sud de la Syrie jusqu'à l'Arabie. Et l'ensemble Stranbej (Turquie/ France) jouera des chants traditionnels kurdes de diverses régions d'Anatolie. 